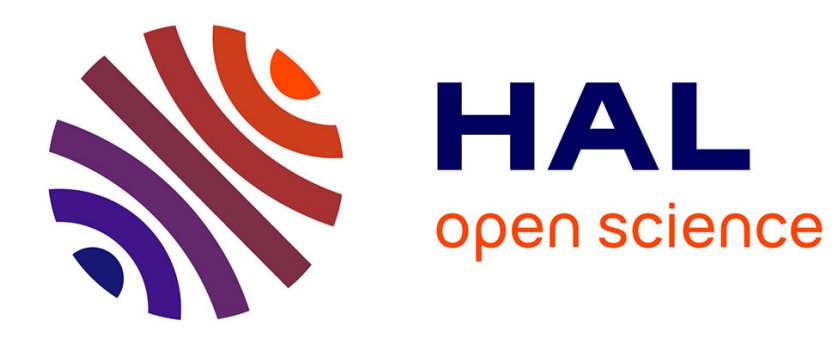

\title{
Le document comme trace ou la trace du document
}

Stéphane Michonneau

\section{To cite this version:}

Stéphane Michonneau. Le document comme trace ou la trace du document. Communications, 2006. hal-01674036

\section{HAL Id: hal-01674036 https://hal.science/hal-01674036}

Submitted on 1 Jan 2018

HAL is a multi-disciplinary open access archive for the deposit and dissemination of scientific research documents, whether they are published or not. The documents may come from teaching and research institutions in France or abroad, or from public or private research centers.
L'archive ouverte pluridisciplinaire HAL, est destinée au dépôt et à la diffusion de documents scientifiques de niveau recherche, publiés ou non, émanant des établissements d'enseignement et de recherche français ou étrangers, des laboratoires publics ou privés. 


\title{
Le document comme trace ou la trace du document.
}

\author{
"Qu'entendons-nous en effet par document, \\ sinon une "trace", c'est-à-dire la marque, perceptible \\ aux sens, qu'a laissée un phénomène en lui-même \\ impossible à saisir?" \\ Marc Bloch, Apologie pour l'histoire ou \\ métier d'historien. ${ }^{1}$
}

En décembre 2001, je reçois, par l'intermédiaire d'un collègue, une lettre d'une inconnue. Arlette Roy, c'est le nom de ma correspondante, m'indique être en possession d'un manuscrit retrouvé dans le grenier de la maison de son père, un manuscrit en castillan dont elle ne connaît pas le contenu, ne maîtrisant pas cette langue. Intrigué, j'apprends que le père d'Arlette Roy, Arnold Brémont, pasteur à Dieulefils puis à Oullins dans les années cinquante, a accueilli nombre de réfugiés espagnols : l'un d'entre eux aurait confié au pasteur le manuscrit. Passé le premier étonnement, ma réaction n'est guère positive : pour les historiens de l'Espagne, la guerre civile est l'équivalent du nazisme pour les contemporanéistes allemands ou de la Révolution pour les dixhuitièmistes français : tout semble y converger nécessairement malgré les efforts des chercheurs pour éviter cette sorte de trou noir qui absorbe tout sur son passage. J'ai toujours soigneusement - et peut-être lâchement - évité de m'y laisser entraîner. Et puis, pourquoi ne pas l'avouer?, je ressens un découragement et presque une lassitude devant le flot de témoignages de combattants de la guerre, de militants et d'exilés : ici comme ailleurs, la force du témoignage a imposé sa loi, limitant la liberté d'historiens sans cesse confrontés au souvenir entêtant des survivants.

Mais comment ne pas répondre à cette offre d'archive? Pour chacun de mes confrères historiens, il s'agirait là d'une aubaine. Mais je n'ai pas le goût fétichiste de l'archive qui tourne parfois au culte positiviste d'une source que l'on met résolument à distance de soi. J'ai travaillé jusqu'à présent sur des documents administratifs sales et poussiéreux, dénués du charme que l'on prête aux écrits manuscrits. A la limite, l'archive m'ennuie et comme beaucoup d'historiens, je me méfie des documents d'éclat qui étalent là leur insolente singularité.

Je traîne des pieds, prétendant être absorbé par les affaires courantes de l'université. A contrecoeur, je vais néanmoins voir Arlette Roy. Le manuscrit comprend deux tomes, 505 pages au total. Les couvertures cartonnées de couleur vert anglais sont cousues main, travail artisanal. Le pasteur Brémont est arrivé dans la Drôme en 1958 et le manuscrit est daté de 1957-1958. Qui donc l'aura déposé là? Mme Roy me parle des réfugiés hébergés au presbytère. Elle insiste surtout sur deux personnes : le premier est Pertusa, un ancien ministre de la IIe République qui, passant outre les conseils du pasteur, revint en Espagne où il fut détenu puis fusillé ; le second est un certain Ramos, ami de la famille qui rencontra sa seconde épouse dans les camps de réfugiés français puis qui partit en Amérique. Mais le lien entre ces deux personnes et le manuscrit n'est pas établi. Ce qui m'interroge et me saisit le plus alors, ce sont les raisons qui ont poussé Mme Roy à ne pas jeter ces pages dactyllographiées trouvées au hasard d'un

\footnotetext{
${ }^{1}$ Marc Bloch, Apologie pour l'histoire ou métier d'historien, Paris, Colin, 1949, p.103.
} 
déménagement : son père, dit-elle, avait toujours souhaité publier le manuscrit. Elle servait sa mémoire.

Assis dans le bus qui me ramène dans le centre de Poitiers, je tiens sur mes genoux 44 ans d'oubli. Me voilà dépositaire d'un manuscrit sans lecteur, apparemment. La matérialité irréductible de l'archive s'impose à moi, une poids inerte qui pèse. Il est là, tout entier trace d'un passé disparu, vestige d'une "voix réduite au silence". On se souvient de cette impression quasi physique éprouvée par Michel Foucault devant le manuscrit du parricide Moi, Pierre Rivière qui précèdent le travail et la mise en oeuvre de la matérialité documentaire ${ }^{2}$. Doit-on pour autant penser comme Marc Bloch que cette trace-là est demeurée telle par l'effet du temps, "comme la fougère, pourrie depuis des millénaires, dont l'empreinte subsiste sur le bloc de houille"3? Probablement pas, car ici la trace n'est pas empreinte mais une trace écrite, une image de la mémoire vive, autrement dit déjà une représentation.

Le statut de ce texte m'intrigue : vierge de toute lecture, il ne semble exister qu'à l'état végétatif. Ce n'est pas tout à fait l'une de ces archives, mise en magasin, qui ne demandent pas à l'historien de les raconter : il s'agit d'un texte pensé et organisé pour être lu, livré intentionnellement au public mais qui, manifestement, a raté son but. Il est mort-né.

Je contourne le texte plutôt que de le lire. Le titre, les Satrapes de l'Occident, fleure l'essai politique fastidieux et démonstratif ; la dédicace aux compagnons de la brigade 139 du front de l'Ebre annonce probablement des mémoires d'ancien combattant ; la signature, celle d'Andreu Martí, est l'unique inscription manuscrite du volume ; l'écriture tapuscrite, serrée et précise, n'est pas précipitée. Non, ce n'est pas une écriture d'urgence écrite sous le feu des bombes mais une écriture appliquée et déterminée, presque implacable. "Hostalric, 1957. Gerona, 1958" lit-on au-dessus de la signature, soit vingt ans après les faits. Prise de parole tardive, lorsque les faits et les dates s'estompent et que ne restent que le souvenir douloureux, stable, presque palpable dans la conscience individuelle. La mention d'une rédaction effectuée en Espagne est étrange car les pages de papier jauni sont écrites au revers de factures d'une blanchisserie... uruguayenne : hasard de la pénurie de matière première dans l'Espagne des années 50? Preuve d'une rédaction en exil? Déjà, un fil invisible est tendu entre Andreu Martí et moi. Rencontre fortuite de deux inconnus qu'il faut se résoudre à accepter, coûte que coûte. Ce lien ténu que je n'ai pas choisi est mémoire. Le manuscrit, tel une bouteille lancée à la mer, m'est revenu si longtemps après : son appel est irrésistible, inévitable. Refuse-t-on une voix qui vous interpelle? Je n'ai pas le choix.

\section{Mémoire oblige}

Le manuscrit est un roman, un récit sans plan, sans structure, sans parties. Il raconte le séjour d'un prisonnier dans les geôles franquistes de 1939 à 1945 puis sa libération conditionnelle, à Tarragone puis à Barcelone, et enfin, la fuite en France. Les premières pages sont une description poignante de l'entrée en prison, un soir de décembre 1939 : abattu, le personnage, Losada, se souvient des derniers combats livrés contre les troupes nationales, l'incarcération dans un camp de concentration au Pays basque, son retour en

\footnotetext{
${ }^{2}$ Michel Foucault, Moi, Pierre Rivière, ayant égorgé ma mère, ma soeur et mon frère..., Paris, Gallimard, Archives, 1973, présentation.

${ }^{3}$ Marc Bloch, Apologie pour l'histoire ou métier d'historien, Paris, Colin, 1949, p.103.
} 
Catalogne où il vécut avant-guerre, son arrestation à Barcelone, l'interrogatoire et les tortures infligées dans un garage puis une usine désaffectée de la banlieue, son voyage en train vers la prison de Pilatos à Tarragone entre deux Gardes civils. Le lecteur plonge sans ménagement dans le cauchemar des pièces obscures et humides, bondées, où l'attente interminable des prisonniers, la peur au ventre, n'est interrompue que par les salves des exécutions, au lever du soleil, les sacas. L'unique humanité qui subsiste, c'est celle que Losada sauvergarde avec les amis d'infortune qu'il ne tarde pas à rencontrer : Serradell, un ancien professeur de mathématique devenu journaliste, Fermin, un ingénieur tarragonais qui a perdu son épouse pendant le conflit et qui reste sans nouvelle de son fils, Masfort, avocat et industriel qui milita dans un parti de la gauche catalaniste, Salgado, ancien champion de natation, étudiant en médecine dont le corps, couvert de cicatrices, est un "vrai champ d'opération", etc. Au quotidien, des stratégies de survie s'élaborent contre un régime carcéral oppresseur, destructeur même. Quatre cents pages d'attente, sans intrigue, comme un long plan-séquence. Le temps paraît comme suspendu, à peine troublé par quelques anecdotes sans grande importance, histoires pour tuer le temps. C'est un manuscrit tissé de non-événements, d'une pure quotidiennité qui restitue l'attente infinie, le vide d'années passées entre quatre murs suintant l'humidité, une pure durée ponctuée par le surgissement irrégulier de la violence et de la mort. La tension entre le temps vide de la routine et le temps court des exécutions sommaires est la véritable respiration de ce texte.

Ce document est celui d'une victime de l'un des pires systèmes concentrationnaires que le XXè siècle ait connu. S'il fallait le classer, il appartiendrait probablement au genre de la littérature concentrationnaire où des paroles gagnées sur l'ennemi et la mort expriment un combat farouche mené contre l'annéantissement ${ }^{4}$. Comment réduire un témoignage de camp à un simple document historique? C'est un témoignage d'un homme qui ne fut pas spectateur mais acteur du drame ${ }^{5}$. Je devine que pour l'auteur, l'écriture permit une seconde naissance : réconciliation avec un passé qui hantait son présent, travail de deuil mais aussi "reconquête de soi"6.

La transmission de la mémoire s'accompagne ici d'un devoir de justice envers les victimes : une nuit, l'un des personnages du roman accompagne les derniers instants d'un condamné à mort. A l'aube, il jure de transmettre la dernière lettre du prisonnier qui dénonce la trahison dont il fut le jouet. Mais le compagnon est fusillé le même jour, anihilant la mémoire et du même coup, l'espoir de justice. Tout se passe comme si l'auteur avait voulu exorciser la perspective de cet engloutissement. Tout entier tendu vers la mémoire, il en oublie la vie d'avant, la Belle Epoque de la République, et la vie d'après, celle de l'exil en France. Les personnages sont comme suspendus dans le temps et seul compte finalement ce moment où leur destin individuel a coincidé avec le destin collectif. L'avant et l'après, renvoyés au domaine privé, sont oubliés pour que ne subsiste que le pendant qui a valeur pour l'histoire, en tant que témoignage dont la portée se veut générale ${ }^{7}$.

La mémoire n'est plus seulement une capacité de se souvenir du passé mais elle est aussi une promesse pour l'avenir entièrement sous-tendue par un exigeant "Plus jamais!".

\footnotetext{
${ }^{4}$ Alain Brossat, L'écriture du désastre, Paris, Albin Michel, 1993, pp. 237 sq.

${ }^{5}$ Paul Ricoeur, La mémoire, l'histoire, l'oubli, Paris, Le Seuil, 2000, p.223.

${ }^{6}$ Michael Pollak, "La gestion de l'indicible", in Une identité blessée, Paris, Métalié, 1993, pp. 140-147.

${ }^{7}$ Michael Pollak, "L'expérience concentrationnaire", in Une identité blessée, Paris, Métalié, 1993, pp.149175.
} 
Dans la mesure où le jugement moral est partie intégrante du processus de compréhension historique, la publication d'un tel récit s'inscrirait nécessairement dans l'horizon de la condamnation d'un tel univers concentrationnaire. J'étais dans la position de ce compagnon de cellule qui avait juré de transmettre la lettre du condamné. Avais-je dès lors des chances d'échapper à la clameur insistante du devoir de mémoire?

\section{S'agit-il bien d'un témoignage?}

En histoire, il n'y a pas de documents exceptionnels, seulement des documents singuliers ${ }^{8}$. Singulier, celui-ci l'est à bien des égards : d'abord, par sa précocité. Rédigé à la fin des années 50, dans un contexte saturé des souvenirs de la Guerre civile, mais uniquement ceux du camp franquiste, il est bien différent des témoignages et des interviews qui abondent ces derniers temps ${ }^{9}$. Ensuite, par sa forme romanesque : l'auteur a voulu délibérément éviter le témoignage direct et l'ancrage particulier qu'il suppose. Pourquoi un roman et non des mémoires?

Je trouve ce choix courageux : le parti pris du roman brouille la frontière entre la part de l'imagination composée d'objets fictifs et la part du souvenir composé des choses du passé bien réelles. Comme historien, il me faut distinguer le fait d'imagination pure, l'invention radicale d'une fiction, et la combinaison des souvenirs réels qui jouent de leur libre association dans le récit, tout en comprenant que cette combinaison a un sens et une vérité, en tant qu'effort de fidélité pour "donner à voir" ce que fut la prison. Mais il y a une différence de taille entre l'auteur qui se souvient et moi, lecteur, qui imagine ses souvenirs sans avoir connu d'expérience carcérale. L'auteur tient le pari de la "résurrection" du passé par la mise en image au risque de voir reléguer son roman au rang de la pure fiction, d'être soupçonné et discrédité.

Ce risque, je tâche d'en comprendre la valeur : l'auteur se situe aux limites de l'intransmissible et aucune mise en intrigue n'était moralement acceptable à ses yeux. Mais il le tente, quite à minorer la portée de son écrit, parfaitement conscient de l'inadéquation entre l'écriture de l'horreur et l'horreur elle-même. En même temps, la forme romanesque ne permettait-elle pas à l'auteur de maintenir à distance des souvenirs difficiles? A dix ans à peine de sa libération, l'autobiographie était-elle tout simplement concevable?

En vérité, je suis bien en peine de qualifier le genre auquel appartient les Satrapes de l'Occident. En effet, l'auteur a brouillé les pistes : le manuscrit comprend deux parties inégales. La première est un récit romanesque qui termine sur le franchissement de la frontière des deux héros Losada et Serradell. La seconde est un essai politique d'une vingtaine de pages, des réflexions que Losada récupéra des mains de Serradell lorsque celui-ci succomba sous les balles des Gardes Civiles frontaliers.

Pour justifier ce collage, Andreu Martí explique que Losada, une fois à Perpignan, lui fit transmettre l'essai en Espagne par l'intermédiaire d'un compagnon d'infortune appelé Bruch. Ce dernier ajouta à l'essai ces propres notes rédigées en prison afin que Martí

\footnotetext{
${ }^{8}$ Annick Lempérière, "Moi, Rigoberta Menchú. Témoignage d'une indienne internationale", Communication, 71, 2001, pp.396-434.

${ }^{9}$ Sur ce point, voir le receuil d'article récent : Antonio Castillo, Feliciano Montero (dir.), Franquismo y memoria popular. Escrituras, voces y represnetaciones, Madrid, Siete Matres editores, 2003, 284p.
} 
rédige une seule histoire à partir de ces documents ${ }^{10}$. "Un jour, tu pourras m'envoyer cette histoire bien arrangée et ordonnée et je me chargerai de la publier hors d'Espagne (...)" commente Bruch à Martí1 ${ }^{11}$.

"Je m'étais déjà rendu compte en commençant par les écrits de Bruch de l'immense responsabilité qui pesait sur moi (...). Bruch connaissait mon goût pour la littérature mais aimer la littérature est une chose, être écrivain en est une autre" confie alors Martí $^{12}$. Les pages de Seradell sont déchirées, maculées, manquantes pour les trois quarts. Dans l'incapacité de réaliser une synthèse acceptable, l'auteur présumé se résoud au collage ${ }^{13}$.

Le manuscrit de Poitiers se termine sur ces lignes : "Songeur, distrait par le souvenir de Serradell, je m'amuser à serrer le bloc de page entre mes doigts. Je pensais à la destinée qu'avaient eu les écrits de mon ami et la forme étrange sous laquelle ils verraient le jour, si l'on pouvait publier l'histoire de Bruch. Je pensais à la petite partie que j'avais pu sauver de tout son travail. Quand je me réveillais, je contemplais sur la table un tas brunâtre formé d'étranges cendres"14.

Cette surprenante conclusion montre que la mention de "roman" indiquée sous le titre est trompeuse : elle cache un jounal et un essai. Mais chacune de ses pièces appartiendrait à un auteur différent, écrivant à des époques distinctes. Le télescopage des formes discursives et des auteurs trouve finalement son unité dans une forme romanesque qui se dénonce à la fin comme telle : le narrateur omniscient sort des coulisses pour expliquer son incapacité à fondre dans un tout cohérent les pièces qui lui sont soumises et ses propres souvenirs. C'est bien finalement la destruction du temps qui commande le collage quelque peu maladroit, le devoir de mémoire vis-à-vis d'un mort et l'urgence à lutter contre l'effacement inéluctable des traces.

La structure en poupée russe m'interdit de trancher sur le statut du témoin : qui parle? Bruch? Martí? Serradell? Et si le véritable auteur n'était aucun de ces personnages, à quel jeu étrange a-t-il voulu convier le lecteur? La mise en scène de l'acte d'écriture au sein même du récit est-elle une coquetterie d'auteur? la maladresse d'un littérateur amateur? un artifice littéraire à la manière du XVIIIè siècle? Un remord ou un doute touchant au choix de la forme romanesque pour porter témoignage?

Ces quelques réflexions me conduisent à ne pas publier le manuscrit sans démêler l'écheveau. "Livrer" un témoignage dont je ne peux d'aucune manière m'assurer du statut? En historien, j'affirme mon droit à ne pas céder au témoin, tout en respectant les trois horizons de son témoignage : le récit d'une vie, l'administration d'une preuve et la fidélité aux morts.

Plus je lis et relis le manuscrit, plus je suis résolu à ne pas céder au "pacte compassionnel" que le témoignage tente de m'extorquer ${ }^{15}$. S'il est bien vrai que la parole

\footnotetext{
${ }^{10}$ Andreu Martí, Los Satrapas en Occidente, s.e., manuscrit inédit, p.480.

${ }^{11}$ Idem, p. 477 .

${ }^{12}$ Idem, p.480, 487-488.

${ }^{13}$ Idem, p. 498-499.

${ }^{14}$ Idem, p.505.

${ }^{15}$ Annette Wieviorka, L'ère du témoin, Paris, Plon, 1998, p.179.
} 
des victimes, des sans-voix et des exclus est un indice de la démocratisation du témoignage, le risque de substitution du témoignage à l'histoire est ici trop important.

\section{Un document d'histoire}

Il n'est pas inutile de revenir ici à l'historien aux archives. C'est armé de questions qu'on va au document, sans quoi ce dernier est muet ${ }^{16}$. Peut-on appeler recherche une étude qui s'en tiendrait à la source telle qu'il la reçoit, qui s'en tiendrait aux limites posées par la source elle-même? Face à l'archive, il faut bien opérer "une redistribution de l'espace" qui permette de définir un lieu où se déploie l'interrogation de l'historien ${ }^{17}$. Ce n'est pas sans violence que je me confronte à ce manuscrit qui semble me dicter sa loi. Que faire?

D'ordinaire, l'historien fait le document "en allongeant le questionnaire" ${ }^{18}$ : le renouvellement des paradigmes aboutit en quelque sorte à une forme d'accumulation des connaissances historiques en ce qu'il porte incessamment à l'archive de nouveaux éclairages. Instituer le document par le questionnement, c'est bien circonscrire ces pages qui ne sont pas un simple donné contenant je ne sais quelle vérité à révéler. Le questionnement maintient donc le témoignage dans un état de suspicion en refusant de prendre pour argent comptant ce qu'il remémore. L'écriture de l'histoire est alors forcément ambiguë : elle est la condition sine qua non de la transmission de la mémoire en même temps que son inéluctable trahison.

Ce problème de la mémoire n'était pas pour moi inédit ; j'avais été nourri à l'école du soupçon car plusieurs années durant, j'avais tenté d'objectiver la mémoire pour l'étudier. Sur un terrain spécifique, celui de Barcelone entre 1850 et 1930, j'avais montré combien la mémoire était une parole sur le passé indissociable d'une pratique sociale, sous forme de cérémonies de remémoration collective inscrites dans une société donnée ${ }^{19}$. Socialement et historiquement déterminée par la société qui la produit dans le présent, la mémoire pouvait être étudiée à froid : qui sont les promoteurs de mémoire? Pourquoi s'arrogent-ils le droit de dire le passé de la communauté? Quel est leur public? Comment se structure la "société commémorante", cette partie de la société barcelonaise qui s'adonne à l'exercice du souvenir? Quelles sont les stratégies par lesquelles les souvenirs de ces groupes actifs se convertissent en mémoire à prétention collective pour la communauté qu'ils entendent représenter? etc.

L'une des principales questions fut de comprendre la valeur sociale de la mémoire. Comme on sait, l'hommage aux ancêtres se présente toujours comme le paiement d'une dette ancienne qui engage toute la société et l'incite à reconnaître un devoir de mémoire. Que signifiait cette obligation du souvenir d'un point de vue politique et social? Les discours des promoteurs de mémoire s'efforçaient de mettre au jour une dette que la société aurait contactée mais dont elle était oublieuse. Le devoir de mémoire, présenté comme impératif et positif, érigait le sacrifice de l'ancêtre en acte inégalable et exigeait en contrepartie l'érection d'un monument, instaurant une véritable économie commémorative. Il était utile de se demander pourquoi une société déterminée, à un

\footnotetext{
${ }^{16}$ Antoine Prost, Douze leçons sur l'Histoire, Paris, Le Seuil, Point, 1996, p. 79. "c'est la question qui construit l'objet historique en procédant à un découpage original dans l'univers sans limite des faits et des documents possibles"

${ }^{17}$ Michel de Certeau, L'écriture de l'histoire, Paris, Gallimard, Folio, 1975, p.100.

${ }^{18}$ Paul Veyne, Comment écrit-on l'histoire?, Paris, Le Seuil, Point, 1971, p.141 sq.

${ }^{19}$ Stéphane Michoneau; Barcelona, memoria i identitat, Barcelona, Eumo, 2002, 429p.
} 
moment donné, établissait la croyance d'un échange avec les morts quand celui-ci n'a aucune réalité? A quoi servait donc l'inculcation d'un devoir mémoire?

L'hypothèse que j'ai avancée est la suivante : la société est bien, in fine, l'unique instance susceptible de libérer les commémorants de la dette qu'ils croient avoir contracté avec le passé. Commémorer, c'est donc toujours se montrer à soi et aux autres comme bon payeur et par conséquent bon citoyen, attentif à ses devoirs. C'est obtenir du crédit auprès de ses concitoyens, de la réputation, du prestige reconnus par tous. L'inculcation de la dette offre donc de nombreux bénéfices à ses promoteurs qui sauront en tirer profit, notamment politique, en s'imposant comme les représentants naturels d'une communauté soit-disant oublieuse d'elle-même. A l'autre bout de l'échelle des valeurs sociales, le public qui refuse l'exercice de la mémoire endosse un rôle qui le discrédite. Pour se libérer de cette forme subtile de domination sociale, il doit obeir au devoir de mémoire : en se mettant en règle avec la société, il n'a pas gagné la protection des ancêtres qu'on lui prométait, mais il a imposé le respect de ses contemporains. Voilà l'un des secrets du dynamisme des sociétés nationalistes que j'étudiais. Finalement, le devoir de mémoire est un système de domination qui permet de hiérarchiser la société de manière subtile. Il contribue à construire la position dominante des élites, notamment intellectuelles, et éclaire d'un jour nouveau le fonctionnement des systèmes politiques représentatifs.

C'est armé de ces questionnements et de ces hypothèses que j'aborde Les Satrapes. La distance qui me sépare à présent d'une approche subjectiviste du manuscrit de Poitiers est immense. Je perçois confusément une sorte de décalage entre ces questions et la nature du document interrogé. Mais mon métier d'historien commande de comprendre le contexte de production d'un tel document : qui est l'auteur? Quelle est l'histoire du manuscrit? Qui sont les personnes réelles qui se cachent derrière les nombreux personnages du roman historique? Il commande également de comprendre les conditions sociales de la prise d'écriture qui permit à l'auteur de s'instituer représentant du groupe des détenus, porte-parole d'une cause. Retracer l'histoire de cette petite intelligentsia républicaine qui crut nécessaire de continuer le combat contre le franquisme, à rebours de la société dans laquelle ils survivaient.

\section{Le document sous le feu de la critique}

L'enquête commence lentement, péniblement. Les indices dont je dispose sont ténus. Renseignements pris, le manuscrit n'a jamais été publié. L'auteur est inconnu : je pressens qu'il s'agit d'un pseudonyme. Par chance, je retrouve l'ultime témoin vivant de la prison de Pilatos : c'est un monsieur âgé, ancien militant et journaliste, plus tard élu sénateur du $\mathrm{PSOE}^{20}$. Il atteste les faits et reconnaît quelques uns des personnages, mais ni Losada, ni ses compagnons. Il m'offre de manière inespérée le radeau auquel ma raison critique peut enfin s'accrocher : le témoignage semble donc fiable. En fait, le témoignage du sénateur sert de reconnaissance de conformité entre le souvenir présent et l'impression première qui ne m'appartient pas, car je suis né 30 ans après les faits. "Le petit miracle de la remémoration", comme dirait Paul Ricoeur, qui rend crédible ce passé vécu, est indispensable à mon travail qui permet de restituer l'objet "au royaume du passé révolu" car je sais désormais qu'il ne s'agit pas de purs fantasmes fictionnels.

\footnotetext{
${ }^{20}$ Josep Subirats Piñana, Pilatos 1939-1941, Madrid, Pablo Iglesias, 1993, 243p.
} 
Mais il est troublant de penser que l'essentiel de cette attestation m'échappe car je n'ai pas vécu dans cette prison. De quelle vérité des faits s'agit-il? La vérité du témoignage repose toujours in fine sur un acte de foi : le lecteur abandonne toute réticence et accepte de croire un témoin qui y était et clame que les choses se sont bien passées ainsi ${ }^{21}$. Quelle est dans tout cela la part de vérité à débusquer? Et la part d'inexactitude, voire de mensonge à indentifier?

La recherche s'intensifie. Le sénateur du PSOE a reconnu l'identité de Salgado, le champion de natation ami de Losada. Je l'appelle pour mettre un nom réel sur le héros principal. Trop tard, ce monsieur est atteint de la maladie d'Heilzeimer. Je cherche Andreu Martí, - pour ainsi dire un Jean Dupont catalan! -, dans les annuaires de Gérone et d'Hostalric des années 50. Sans résultat. Dans les mairies, le cadastre ne donne rien non plus. Je décide de suivre la piste des deux amis du pasteur Brémond : pour confier le manuscrit au pasteur, il fallait qu'il existât un lien d'amitié entre les deux hommes, c'est du moins l'hypothèse que je formule. Pour le ministre Pertusa, fusillé dès son entrée sur le territoire espagnol en 1944, comment aurait-il pu confier un manuscrit daté de 1957-1958? Reste Antonio Ramos. Il est arrivé en France avec un petit garçon, me précise Arlette Roy : cela me rappelle le personnage Fermin du roman. Il a connu en France Pilar Moll, elle-même réfugiée avec un enfant. Il a connu le pasteur en 1950 à Oullins, dans le Rhône, qui l'a marié à Pilar. Puis, la nouvelle famille serait partie au Brésil la même année. Arlette Roy consulte le carnet d'adresse de sa mère : il s'agit de l'Uruguay, pas du Brésil! Ils sont revenus en France deux fois, en décembre 1963 puis en avril 1964. Antonio Ramos est mort en Espagne en 1972, peut-être à Barcelone. Son épouse y vivait encore en 1983, date du dernier agenda consultable. A priori, ces faits sont contradictoires avec la mention "Hostalric 1959, Gérone 1960". Si les Ramos sont entrés en Espagne, c'est probablement en 1969, date de l'amnistie pour les exilés politiques. Mais le détail de l'Uruguay m'intrigue : expliquerait-il le revers des factures?

Dans l'annuaire de Montevideo, il n'y a plus de Ramos à l'adresse notée par l'épouse du pasteur Brémond en 1950. Je cherche son fils en vain : il existe des milliers de Ramos en Uruguay. Il me faudrait le nom de la mère puisque les Uruguayens, comme les Espagnols, portent les noms de leurs deux parents, mais je ne l'ai pas. Qui me dit d'ailleurs que son fils est resté en Uruguay? Et son beau-fils? D'après Arlette Roy, il est rentré en Europe et vivait à Zurich dans les années 80 . Je ne le retrouve pas : cette foisci, j'ai bien le nom de sa mère mais pas celui de son père. Je cherche à Barcelone la trace de Pilar Moll, en vain. Je cherche dans les archives françaises la trace des Ramos: le registre d'entrée des immigrants de l'Office des Migrations Internationales a brûlé récemment. Il me faut aller aux archives préfectorales du Rhône et de la Drôme. Mais les sorties du territoire français ne sont pas indiquées. Il me faudrait trouver les noms des enfants : l'aîné avait une dizaine d'année en 1950, une soixantaine aujourd'hui...

En attendant, je cherche les archives de la prison de Pilatos : j'apprends qu'elles sont heureusement conservées à Tarragone. Cependant, s'agissant d'informations touchant des personnes possiblement vivantes, elles sont inaccessibles. Je tente le tout pour le tout : l'archiviste est un fonctionnaire très fier de son pouvoir discrétionnaire. Après de longs palabres, j'ai le droit de jeter un oeil sur le registre des prisonniers. Il y a un Andreu Martí (y Finells) et un Antonio Ramos (y Sánchez). La piste est sérieuse mais je ne peux consulter leurs dossiers.

\footnotetext{
${ }^{21}$ Paul Ricoeur, La mémoire, l'histoire..., Op. Cit., p.?
} 
Pour contourner l'obstacle, j'écris aux archives militaires de Barcelone qui, en principe, conserve les dossiers d'inculpation. L'archiviste s'avère très compréhensif. Après plusieurs semaines, j'accède finalement aux dossiers. Celui d'Antonio Ramos est intéressant ${ }^{22}$.

Né à Madrid (comme Arlette Roy le disait) en 1909, il est emprisonné à Tarragone en décembre 1939, comme Losada. Il passe au conseil de guerre le 28 juin 1940, ce qui correspond à la date que j'ai pu reconstituer à partir des indices du roman et des listes publiées dans la presse en 1940. A partir de 1935, il est un maître d'école dans le sud de la Catalogne, près de Tortosa (Arlette Roy m'avait parlé d'un professeur de mathématique à L'Université de Madrid). Il a bien un fils, comme Fermin dans le roman. Dans l'acte d'accusation du Tribunal Militaire, il est jugé pour "rébellion militaire en tant qu'officier de l'armée républicaine", dans le quatrième bataillon, brigade 139 : celle de la dédicace! Il fut fait prisonnier en rentrant en Espagne après un court exil français puis déporté dans un camp de concentration au Pays Basque, comme Losada. A quelques informations près, la concordance entre Ramos et Losada saute aux yeux. Il me faut en avoir le cœur net.

Retrouver un anonyme balloté par la guerre, la répression et l'exil n'est pas chose facile. Antonio Ramos me fait penser à ces "hommes infâmes" dont Foucault aimait à retracer l'existence ${ }^{23}$. Il me faut absolument accéder aux archives de la prison de Tarragone, bien entendu, mais d'autres pistes s'ouvrent : les archives du ministère de l'Education Nationale, transférées à Barcelone depuis la naissance des Autonomies, celle du Patronato de Rendención de las Penas, à Madrid, celles des officiers de l'armée républicaine, à Avila. A Madrid, à Tortosa, et à Barcelone, je cherche les dates de naissance et de décès d'Antonio Ramos et de sa première épouse dont j'ai trouvé le nom dans les archives militaires. J'écris à Tortosa pour retrouver sa trace et l'acte de naissance de son fils : dans le roman, le fils de Fermin à sept ans. Il me faut accéder aux archives des camps de concentration de Bilbao. J'obtiens l'aide de l'Association des Ex Prisonniers Catalans. Et puisque Losada est protestant dans le roman, une rareté en Espagne, je mobilise les réseaux de l'Eglise Evangélique de Catalogne... mais ne seraitce pas plutôt une forme d'hommage qu'Antonio Ramos aurait rendu a posteriori à son ami, le pasteur Brémond? Cela signifie-t-il que le manuscrit fut rédigé en partie en France, et en Uruguay?

Quelle est la valeur de ces va-et-vient continuels entre la réalité et la fiction? J'oscille insensiblement entre plusieurs niveaux de réalité, m'épaulant sur l'un pour comprendre l'autre. Pour le moment, cela m'a porté chance... A présent, j'ai bon espoir de reconstituer la vie de l'auteur et l'histoire du manuscrit. Peut-être même, grâce aux dossiers militaires de la prison, celle de certains des personnages du roman...

\section{La mise en mémoire, dans les années 50.}

Reste à résoudre le difficile problème du contexte de la mise en mémoire. Il me faut expliquer d'abord pourquoi Ramos a du écrire. En effet, le contexte espagnol d'aprèsguerre aurait pu vouer une victime de la répression au silence et à la culpabilisation. Par

\footnotetext{
${ }^{22}$ Arxiu de la Diputació de Tarragona et Arxiu Militar Territorial III; Barcelona, $\mathrm{n}^{\circ} 42526$, procediment $\mathrm{n}^{\circ}$ SU 4217.

${ }^{23}$ Michel Foucault, "La vie des hommes infâmes", in Dits et écrits II, Paris, Gallimard, pp. 237-253.
} 
ailleurs, comme on a vu, j'ai des raisons de penser que ce document a été partiellement rédigé hors d'Espagne. J'imagine Ramos, exilé en France aux lendemains de la guerre, à l'image de ces centaines de milliers d'Espagnols qu'a décrit Geneviève DreyfusArmand $^{24}$, lui, l'ancien instituteur devenu garagiste à Oullins comme Arlette Roy me le précise maintenant. Est-ce à la suite de difficultés d'intégration en France qu'il partit en Uruguay avec sa nouvelle famille? Michel Pollak a remarqué que l'écriture et la remémoration permettaient au témoin de surmonter une crise conjoncturelle ${ }^{25}$ : dans ce cas, l'exil entraina probablement une rupture qui motiva l'acte d'écriture. Bien souvent aussi, Pollak a décelé l'encouragement d'un homme religieux à l'origine de la mise en mémoire: ici, le mariage de Ramos célébré par le pasteur Brémond présida peut-être une conversion religieuse, laquelle, en offrant une nouvelle socialisation, coupa cependant davantage Ramos de sa vie antérieure. Ainsi, l'impossibilité d'articuler sa mémoire individuelle à la mémoire collective des autres exilés pourrait expliquer la nécessité de la prise de parole. En relatant ces temps forts où les raisons générales de la lutte contre le franquisme se confondaient avec les motivations intimes de résister, Ramos rétablissait une certaine cohérence perdue dans l'exil. Par l'évocation de ses amis si chers, il reconstituait ce cercle de proches nécessaire à l'évocation première du souvenir, cette fraternité qui l'avait sauvé mais qui, en s'évanouissant, l'avait conduit à l'isolement.

En somme, Ramos avait connu un double exil : sorti de la prison de Pilatos et des camps de travail en 1945, son personnage Losada végète à Barcelone en paria de la nouvelle société franquiste : difficultés de ravitaillement, chomage entrecoupés de courtes périodes d'embauche en tant qu'ouvrier, humiliations répétées devant des autorités revanchardes qui le surveillent étroitement, auprès du curé qui refuse de délivrer un certificat de bonne conduite, échecs réitérés de tentatives d'émigration légale auprès des consultats sud-américains, résistance passive sous l'oeil de la police secrète... "L'Espagne était une immense prison" conclut-il. Si le roman se termine sur une note d'espoir, le passage de la frontière, Losada y perd son ami, une part de lui-même. Le prix de la liberté fut l'expérience d'une désocialisation que seul le roman publié pouvait conjurer.

J'ai donc bien affaire à un entrepreneur de mémoire s'arrogeant un devoir sacré au nom d'une vérité qu'il défend avec intransigeance, une vérité élaborée dans les années 1950. En effet, Ramos manifeste vis-à-vis du communisme une haine inextinguible car pour lui, la démocratie espagnole fut perdue par la victoire des totalitarismes fasciste et communiste. En 1939, par le pacte germano-soviétique. En 1946 par la reconvertion du franquisme en digue de l'anticommunisme. L'opinion est assez caractéristique du militantisme socialiste et républicain (pendant la guerre, Ramos était affilié à l'UGT) et reflète parfaitement les profondes divisions des gauches espagnoles pendant et longtemps après le conflit. En même temps, l'obsession antitotalitaire traverse l'ensemble du manuscrit émaillé de nombreux anachronismes : par exemple, depuis sa prison catalane entre 1939 et 1943, Losada parle d'Auschwitz. Plus loin, il parle des camps staliniens en des termes qui sont postérieurs à la déstalinisation. Les Satrapes est un écho d'une conscience choquée de l'après-Seconde Guerre Mondiale.

Il faut également rappeler le contexte espagnol que l'auteur, récemment éxilé, ne pouvait ignorer. Les années 50 marquent la fin du franquisme totalitaire et la poussée

\footnotetext{
${ }^{24}$ Geneviève Dreyfus-Armand, $L$ 'exil des républicains espagnols,

${ }^{25}$ Mikael Pollak, Nathalie Heinich, "Le témoignage", Actes de la Recherche en Sciences Sociales, 62-63, 1986, pp. 3-29.
} 
d'une nouvelle génération dirigeante réformiste proche de l'Opus Dei. Ce virage définitivement opéré en 1960 changea le visage de la dictature et permit sa survie. Dès lors que le régime paraissait installé pour longtemps, le souvenir de la guerre civile s'estompa au sens où le régime pouvait désormais compter sur d'autres formes de légitimation que furent la paix et la prospérité économique. Je peux comprendre ce qu'avait d'impérative la dénonciation du "dernier régime fasciste d'Europe" pour un Ramos qui voyait partir en fumée les ultimes espoirs d'un retour à la démocratie : son départ en Uruguay dut ne pas être étranger à ce constat désespérant.

Les années 50 marquent aussi les débuts d'une constestation sociale : la séquence de grèves commencée à Barcelone en 1951 connut son apogée avec les événements estudiantins de 1956, à Madrid, premiers actes d'une protestation sociale qui mobilisa les milieux ouvriers ${ }^{26}$. C'est dans ces luttes que devaient se former les élites de la résistance à la dictature tout au long des années 60 et 70 . Cependant, le front contestataire était formé des fils "de vaincus et de vainqueurs", déçus par l'absence de perspective que leur offrait le régime. Cette nouvelle génération qui n'avait pas connu les combats de la guerre choisit de se tourner résolument vers l'avenir, sans plus évoquer les divisions du passé. De sorte que le renouveau espagnol passait par la négation de l'expérience des anciens combattants et des victimes de la répression ${ }^{27}$. Dans ce pays qui s'aprêtait à tourner la page de la guerre, Les Satrapes revendiquait haut et fort l'actualité de son combat.

Le contexte de production de ce manuscrit est aussi le destin effroyable de cette petite intelligentsia républicaine, faite d'instituteurs, de professeurs, d'employés, de journalistes, de médecins, d'avocats, etc., littéralement broyée par le nouveau régime. Autant que la classe ouvrière, le franquisme s'employa avec une brutalité inouïe à éradiquer la présence sociale de ces cadres qui représentaient l'unique espoir de reconstruire un autre monde. Cette Espagne moyenne, nourrie d'une tradition libérale séculaire et volontiers ouverte à l'avant-garde culturelle, fut extirpée comme on arrache une mauvaise dent, sans anestésie. La mémoire de ces groupes isolés et martyrisés se heurte à celle du régime mais aussi à celle des générations d'après-guerre. Si la mémoire se décline sur le mode du conflit, le Ramos des années 50 est un promoteur de mémoire isolé et voué à l'échec.

Ainsi, je dessine les contours de la mise en mémoire à partir des quelques indices dont je dispose. Encore faudra-t-il affiner l'analyse mais lorsque ce travail sera achevé, je pourrai publier le manuscrit, le commenter, le préfacer. Par mes questionnements, le manuscrit sera devenu un document.

\section{Histoire ou trahison?}

Mais document, ne l'était-il pas déjà devenu lorsque le pasteur l'avait sauvé, mis en archive? Par mon travail, je crois restituer la mémoire d'une victime du franquisme, alors que c'est peut-être celle du pasteur Brémond que je sers. En effet, confronté à d'autres témoignages, ce manuscrit deviendra une pièce, un indice supplémentaire dans la construction d'une preuve documentaire. N'est-ce pas trahir Antonio Ramos qui avait

\footnotetext{
${ }^{26}$ Jesús Martínez (dir.), "La consolidación de la dictadura (1951-1959)", in Historia de España, siglo XX, 1939-1996, Madrid, Cátedra, 1999, pp. 106-117.

27 Ponencia de Santos Juliá, "Memoria e identidades", VII Congreso de la Asociación de Historia Contemporánea, Santiago de Compostela, 21-24 de setiembre de 2004. A paraître.
} 
pensé écrire un roman? Traiter ce roman en archive: sacrilège? Transformer le témoin en "document vivant" : indécence ou fidélité à la mémoire de l'auteur? La trahison serait-elle la condition de la survie du document?

J'ai le sentiment que ma démarche froide, distanciée et critique dans l'usage des catégories, des classements, est incapable d'expliquer ou d'atteindre ce que fut l'expérience d'Antonio Ramos, qu'elle le manque au moment où, dans sa conception naïve et appauvrie du rapport au réel et à la mémoire, elle pensait l'avoir compris. N'y at-il pas mieux à faire que de considérer le roman sous l'angle du soupçon pour fauxtémoignage?

On ne peut assumer une critique textuelle historique sans même examiner sérieusement la question de savoir pourquoi certains genres (les mémoires, les témoignages) sont plus historiques que d'autres, plus fiables. Je me trouve dans la position absurde de celui qui tente de retirer d'un récit un "résidu" de vérité qu'il contiendrait, à l'instar des folkloristes du XIXè siècle qui cherchaient dans les chansons de geste et les chansons populaires de leur temps à comprendre la société médiévale ou l'origine de leurs nations. De toute manière, quel est l'instrument qui permettrait de trier l'information vraie de la fausse ${ }^{28}$ ? Certes, Ramos a menti : il n'était pas professeur d'université par exemple. Certes, si je puis dire, Losada ment : Ramos n'a pas combattu les franquistes jusqu'au bout et a séjourné dans les camps de concentration français, comme un demi million de ces compatriotes. C'est en rentrant en Espagne qu'il fut détenu. Et maintenant? Les abus de la mémoire ont fini par rejaillir sur les mémoires individuelles qui se trouvent désormais dénuées de toute crédibilité.

Ce récit n'est rien moins qu'un genre parmi d'autres qui conserve et transmet la mémoire individuelle et sociale. Et la mémoire n'est rien d'autre qu'une tentative pour donner du sens au passé. Il faut donc traiter ce roman historique comme on traiterait une source orale : on ne peut prétendre en retirer ces pépites de vérité qui seraient des grains purs d'information historique, une chose que je ne suis pas en situation de démontrer. Je dois sacrifier l'une des dimensions de la construction documentaire. Le problème n'est pas que le récit conserve de l'information vraie ou fausse mais bien plutôt qu'il conserve de l'information stable d'un point de vue mémoriel, suffisamment pour que l'auteur, vingt ans après les faits, s'en souvienne et les consigne. Finalement, en comparant ce dont se souvient Antonio Ramos en 1960 et ce dont se souvient le sénateur socialiste, on se ferait une idée juste de l'évolution de la perception que le groupe social a de son passé ou bien, du changement de perception de ce dont il est socialement acceptable de se souvenir.

D'autres historiens ont établi un constat de demi-échec de l'approche documentaire. Quelques uns ont tenté d'ouvrir des voies d'un renouvellement de la question $d u$ document. Lorsque Arlette Farge reconstitue la vie de Pierre Le Roy exécuté à Cambrai en 1770, "un personnage qui vit en archive", elle prévient que "ce n'est pas un livre, ni une pièce de théâtre, c'est une forme d'écriture qui raconte et qui expose. Narration, exposition, fiction, histoire, récit (...). Il y eut le désir de prendre le parti de Pierre, ce morceau d'homme qui avait voulu se tailler une trop grande part de responsabilité en

\footnotetext{
${ }^{28}$ Jean Norton Cru, Du témoignage, Op. Cit., p.13. C'est moi qui souligne. Voir également, Christophe Prochasson, "Les mots pour le dire : Jean Norton cru, du témoignage à l'histoire", RHMC, 48-4, oct-déc. 2001, pp. 160-189.
} 
désirant (...) faire ressembler Cambrai à la capitale"29. Le défi consistait à "allier la fabrication du récit historique à la matière de l'histoire". De Louis-François Pinagot, Alain Corbin restitue la vie d'inconnu en s'inspirant de l'intuition de Carlo Ginzburg pour qui l'histoire est un roman qu'on peut prouver. Carles Vinyes dans Le Soldat de Pandore, retrace l'histoire d'un révolutionnaire catalan et finit par "opter pour une structure littéraire qui respecte scrupuleusement autant le contenu du récit oral que les autres sources d'information qui [lui] avaient été utiles soit pour vérifier, soit pour élargir le renseignement" ${ }^{30}$.

Toutes ces tentatives révèlent le même doute : le récit d'historien, composé d'éléments de narration fictive pour donner à voir et à comprendre échappe-t-il si radicalement au soupçon dont le document est entâché? Le retournement du soupçon envers l'historien résulte d'abord de sa présentation littéraire des choses, de sa mise en représentation ensuite où explications et compréhension de l'événement ne préexistent pas à leur mise en intrigue. Il résulte enfin du jugement moral dont l'historien ne peut se départir, quoi qu'il en dise. En somme, le récit historique n'est-il pas trop prompt à jeter le voile du soupçon là où les modalités littéraires qu'il utilise abusent de la confiance du lecteur en confondant la force de persuasion et l'inculcation d'une croyance non fondée? Comme ces historiens, je me méfie des anathèmes d'une histoire universitaire qui a tant de mal à trouver sa place entre science et littérature, entre explication savante et fiction.

En somme, n'y a-t-il pas une manière d'aborder le manuscrit de Poitiers sans le trahir et sans renoncer pour autant à la rigueur critique? Farge, Corbin ou Vinyes tentent de trouver 'les modes de narration propres à avérer les sujets de l'âge démocratique, les modes de subjectivation multiples, pluriels, aléatoires. Trouver une écriture de l'âge des grands nombres, des nouvelles formes de subjectivation, nouvelles émergence de parole, de voix" ${ }^{31}$. Cependant, ils refusent de rompre avec le narrateur omniscient qui se manifeste indirectement, de ce "narrateur en coulisse" comme l'appelle joliment Régine Robin. C'est pourquoi leur mode d'écriture demeure réaliste : il présuppose la transparence d'une écriture transitive, qui n'est pas en elle-même sa propre fin mais qui sert à désigner, à montrer et à juger. En somme, le réalisme est une écriture optimiste dans le sens où le monde qu'elle représente est totalisable : elle est affirmation d'un monde plein.

Parce que la mémoire est absence et disparition, je souhaite trouver une écriture en creux susceptible de rendre compte du monde vide du manque et de l'oubli. Et paradoxalement, cette écriture ne doit pas chercher à éloigner l'historien du document mais au contraire, à l'en rapprocher pour assumer une certaine forme de subjectivité. Sur cette voie, Nicole Lapierre m'a précédé. En cherchant à saisir la mémoire de la communauté juive de Plock, en Pologne, elle se heurte "au paradoxe de (sa) démarche" : "Je sonde le passé pour mieux mesurer ce qui m'en sépare, et prend cette mesure avec des outils intellectuels que cette séparation même m'a pemis d'acquérir" ${ }^{32}$. Consciente que la mémoire est "une boîte noire qui jamais ne livrera son triste secret", elle hésite toutefois à assumer son rôle de passeur, d'intermédiaire qui est "la part d'ombre de (son) personnage d'enquêtrice". Elle demeure comme étrangère, aux frontières d'une histoire possible de la trace documentaire.

\footnotetext{
${ }^{29}$ Arlette Farge, La nuit Blanche, Paris, Le Seuil, 2002, p.6, 9 et 12.

${ }^{30}$ Ricard Vinyes, Le soldat de Pandore, CMCAS de Corse, CCAS, 2004, p.12.

${ }^{31}$ Régine Robin, La mémoire saturée, Paris, Stock, 2004, p.298.

${ }^{32}$ Nicole Lapierre, Le silence de la mémoire, Paris, Le livre de poche, Biblio, 2001, p.302 et 311.
} 


\section{Les mémoires "saturées"}

L'approche documentaire que je cherche ne se propose pas de renouer avec un subjectivisme psychologique, à la manière romantique. Au contraire, elle se propose de prendre en compte la position temporelle qu'occupe l'historien par rapport à son document. Elle pose l'hypothèse que, si le manuscrit fait document, ce n'est pas seulement par les conditions de sa production entre Gérone, Oullins et Montevideo, quelque part à la fin des années 50, mais aussi par les conditions sociales de sa lecture, dans l'Espagne d'aujourd'hui, à l'affût des souvenirs traumatiques des crimes du franquisme.

Ce roman voulait être une preuve judiciaire dans le procès du franquisme que son personnage principal, Losada, ne cesse d'appeler de ses voeux. Confiant dans l'avenir, pouvait-il imaginer que la démocratie espagnole, une fois rétablie, ferait tout son possible pour éviter de traduire en justice les criminels d'autrefois? Trahi par l'historien, le document ne fut-il pas trahi une seconde fois par l'Espagne?

Depuis 1997 environ, le pays est littéralement submergé par un flot de témoignages de la répression franquiste qui n'est pas sans rappeler le surgissement brutale de la mémoire de Vichy en France, au début des années 70. Le soixantième anniversaire du déclenchement du conflit, en 1996, avait permis d'évoquer le souvenir des brigadistes internationaux ${ }^{33}$. Celui de la fin du conflit, en 1999, a mis en lumière l'extrême brutalité du système répressif du franquisme : les fosses communes découvertes de-ci de-là dans d'innombrables villages et dans lesquelles reposent quelques 10000 cadavres patiemment exhumés. Les adoptions illégales des "enfants volés" à leurs parents républicains confiés à des institutions religieuses peu regardantes. Le travail forcé des "esclaves de Franco", ce demi-million de prisonniers républicains qui, dans la centaine de camps de concentration très récemment répertoriés, travaillèrent dans des conditions inhumaines au profit du régime et d'entreprises florissantes. Ceux qui vécurent 40 ans emmurés et qu'on appela "les topos" [de tapar, cacher], pris dans la souricière d'un régime qui ne conçut jamais le moindre pardon sinon l'implacable annéantissement des démocrates. Les femmes, fréquemment violées et battues, dans des prisons dont on ignorait jusqu'à l'existence. Etc, etc...

Pas un jour ne passe sans qu'un article, une recherche, une publication ne dénonce "le pacte de silence et d'oubli" de la démocratie ${ }^{34}$ : la société espagnole aurait été frappée d'amnésie collective, un silence scellé par une classe politique soucieuse de réconcilier les "deux Espagnes". A cet égard, les titres de quelques unes des parutions les plus récentes sur le sujet sont éloquentes : Le passé caché, la mémoire inconfortable, le silence brisé, la voix endormie... ${ }^{35}$. La réussite de la transition dépendait en effet de la

\footnotetext{
${ }^{33}$ Angeles Egido, "Memoria y represión", Historia del presente, la memoria de la segunda República, 22003, pp; 139-147.

${ }^{34}$ Paloma Aguilar, Memoria y olvido de la guerra civil española, Madrid, Alianza editorial, 1996.

${ }^{35}$ En 2002 par exemple, Carlos Elordi présente un programme sur la première radio espagnole en terme d'audience, Cadena Ser, "les années difficiles" : le propos est de divulguer les témoignages, les lettres ou les notes de personnes anonymes victimes de la répression d'après-guerre. De même, Rafael Torres dans Victimas de la Victoria et Rodolfo et Daniel Serrano dans Toda Espana era una carcel s'appuyent sur des témoignages d'ex prisonniers du franquisme. La littérature s'empare du thème comme en témoigne
} 
continuité au pouvoir des élites franquistes converties au pluralisme politique. Dans ces conditions, l'opposition exigea l'amnistie des prisonniers politiques, obtenue en 1977, sans jamais même évoquer la possibilité de constituer une commission de la vérité. L'acceptation de la part des partis de gauche du cadre de la monarchie conservatrice impliqua une renonciation douloureuse aux mythes des deux républiques et à leurs symboles. La démocratie prenait alors le risque de tourner le dos aux seuls expériences démocratiques qu'avait jamais connu le pays.

En fait, la transition a établi l'idée d'une égalité morbide entre les deux camps en conflit $^{36}$. L'idée prédominait d'une lutte fratricide et cruelle de part et d'autre, d'une guerre civile somme toute inutile. Même l'expression de "guerre civile" portait finalement préjudice à la mémoire des combattants qui, d'un côté comme de l'autre, avaient vécu le conflit comme une invasion, une guerre de la véritable Espagne contre l'anti-Espagne. Mais en mettant l'accent sur le coût humain d'une répression qui s'opéra uniquement à l'encontre des vaincus, la distinction entre les victimes de la guerre proprement dite et celles de la répression s'impose désormais. L'assymétrie des comptes a brisé désormais le consensus et le partage de la culpabilité sur lesquels s'est bâtie la transition.

S'il y eut silence, il ne fut assourdissant que de la part de la classe politique et des autorités. Depuis la fin des années 60 déjà, la guerre a accaparé une large partie de l'attention des historiens tant espagnols qu'étrangers, notamment britanniques : on peut parler à cet égard d'un véritable "pacte de la mémoire", à la mesure de l'ouverture des archives. Mais les souvenirs de la génération de la transition furent sélectifs : ceux des combattants de l'intérieur l'emportèrent sur ceux de l'exil, ceux des militants sur celle des victimes anonymes de la répression et de manière générale, ceux de la Guerre sur ceux du franquisme. C'est donc récemment qu'apparaissent des études d'ensemble sur ce phénomène connu mais peu travaillé : Victimes de la guerre civile paru en 1999 sous la direction de Santos Juliá, constitue à ce titre un tournant ${ }^{37}$.

L'avalanche d'expositions, d'émissions de télévision, de publication d'essais a coïncidé avec l'arrivée au pouvoir des conservateurs en $1996^{38}$. Le Parti Populaire ouvrit la voie à une sorte de "révisionnisme" historique qui tâchait de réévaluer positivement la dictature à la lumière, notamment, du miracle économique qu'avait connu le pays. Cette politique se manifesta par le retour d'un nationalisme étroit et la relecture unitaire du passé de l'Espagne. Concrètement, elle justifia le rejet au Parlement de voter la condamnation officielle du coup d'Etat du 18 juillet 1936 jusqu'au 20 novembre 2002, soit 27 ans après la mort de Franco! Pour bon nombre d'Espagnols, cet espagnolisme à

Sefarad de Antonio Muñoz Molina, La voz dormida de Dulce Chacón et surtout Les soldats de Salamine de Javier Cercas (Barcelona, Tusquets, 2001), vendu à plus de 450000 exemplaires.

${ }^{36}$ Paloma Aguilar, "Presencia y ausencia de la guerra civil y del franquismo en la democracia española. Refelxiones en torno a la articulación y ruptura del "pactio de silencio"", in François Godicheau, Historia y memoria de la guerra civil, Actes du coloque de la Casa de Velazquez, à paraître, pp. 106-130. A paraître. Je remercie l'auteur de m'avoir communiqué les actes avant leur parution.

${ }^{37}$ Santos Juliá (coord.), Víctimas de la guerra civil, Madrid, Temas de hoy, 1999.

${ }^{38}$ On peut estimer que la rupture du pacte de silence de la classe politique date des élection de 1993 quand le PSOE, sur le point de perdre le pouvoir, lança une campagne violente contre une droite accusée d'être l'héritière du franquisme. Le PSOE remporta les élections de justesse. 
outrance a marqué un point de non retour : le prix de l'amnistie ne compensait désormais plus le coût de l'amnésie ${ }^{39}$.

La société espagnole est donc entrée brutalement dans l'ère du "tout-mémoire", pour reprendre Pierre Nora. Cette onde de choc, je ne peux prétendre y échapper. Sous la pression d'une société qui exige une expertise, je me trouve, moi et tant d'autres historiens de la guerre civile et du franquisme, dans une situation similaire à celle des historiens français lors des grands procès contre les complices du régime de Vichy ${ }^{40}$, sans compter les effets dévastateurs de la judiciarisation de leus travaux ${ }^{41}$.

Au cours de récents colloques, j'ai pu constater que l'exigence sociale de mémoire divisait les historiens espagnols selon une ligne de fracture générationnelle : l'incompréhension entre "les enfants de la guerre" et "les petits-enfants" rappelle curieusement celle entre les "pères" et les "enfants" à la fin des années 50.

D'un côté, les historiens de la génération des révoltes étudiantes de 1956, principaux artisans du compromis de la transition, rappèlent que la mémoire de la guerre civile servit à faire barrage au maintien d'une l'extrême-droite hostile au processus de démocratisation. L'Eglise avait imposé une lecture des événements en terme moral davantage que politique, la guerre étant le mythe de fondation d'une nouvelle Espagne. La production d'un puissant système d'inhibition sociale conduisit les opposants à choisir alors une stratégie d'approfondissement radical des présupposés idéologiques du régime. Commença alors un long voyage intérieur qui devait amener les enfants des vaincus et des vainqueurs à constater le contraste grandissant entre la perception de la réalité et la mémoire transmise par le régime. De ceci naquit une forme de populisme qui rapprocha les libéraux des secteurs de l'Eglise les plus engagés dans l'action sociale. La guerre apparaissait alors comme "cet inutile combat fratricide" qui n'avait résolu aucun des problèmes qu'elle avait posés. Cette nouvelle mémoire en gestation, activement promue par le Parti Communiste, permit de créer un large front d'opposition qui incluait tous les enfants de la guerre. Naturellement, la transition démocratique héritait de cette mémoire consensuelle.

D'un autre côté, de jeunes historiens défendent la thèse du "pacte d'oubli", bien décidés à rompre ce qu'ils estiment être un tabou de la société espagnole. Nés pendant la transition, le "retour du refoulé" est pour eux un événement fondateur. Leur préoccupation est d'abord le signe d'une transition achevée : le pacte de silence a perdu de son sens maintenant que la démocratie est stabilisée et que l'alternance politique est chose normale (pas tant celle de 1996 que celle de 2004 d'ailleurs). Face au Parti Populaire, elle marque également la renaissance d'une gauche décomplexée et attentive à ses origines : le chef du gouvernement actuel ne s'est-il pas réclamé de son grand-père qui fut officier de l'armée républicaine? Enfin, elle est à l'unisson d'une demande sociale de reconnaissance des victimes, largement relayée par un tissu associatif très mobilisé :

\footnotetext{
${ }^{39}$ Voir Manuel Rodriguez Rivero et Juan Pan-Montojo, Ideologia y cultura en la Espana de los vencedores, Michel Richards, Un tiempo de silencio, la guerra civil y la cultura de la represion en la Espana de Franco, d'Elena San Roman, Francesc Vilanova i Vila-Abadal, Isamel Saz, España contra España, Alberto Gomez Roda, Gilcerio Sanchez Recio.

${ }^{40}$ Henry Rousso, "Juger le passé? Justice et histoire en France", in Vichy, L'événement, la mémoire, l'histoire, Paris, Gallimard, Folio, pp. 678-710.

${ }^{41}$ Paul Alliès, "Les usages politiques de l'histoire en France. La république face au régime de Vichy et à la guerre d'Algérie", Colloque "Histoire et mémoire", Université du Costa Rica, CCCAC, San José, sept. 2003.
} 
Association pour la Récupération de la Mémoire Historique ${ }^{42}$, association des fils d'exilés, association des ex-prisonniers... Ces historiens comprennent mal aujourd'hui que la démocratie ait si longtemps refusé d'assumer la mémoire de la République. Mais la transition n'était-elle pas indissociablement liée à la monarchie? Comme l'a résumé Josep Maria Colomer, cette génération a le sentiment que "les vertus de la transition se sont convertis en vices de la démocratie" ${ }^{43}$.

Comme le remarque Paloma Aguilar, la notion de "pacte d'oubli" participe d'une théorie du complot : par sa rétroactivité, la loi d'Amnistie de 1977 fut avant tout le reflet d'un accord tacite entre les élites politiques pour ne pas instrumentaliser le passé. Mais ce silence ne fut pas uniquement le fruit d'une imposition : la société espagnole dans son ensemble, hantée par la crainte d'un retour de la guerre civile, ne voulut pas réveiller les fantômes de sa mémoire. C'est ce fantôme qui rendit possible le pacte et non l'inverse. De plus, il faut bien reconnaître qu'il existe une différence entre la mémoire de la guerre civile et celle du franquisme, ces deux périodes produisant des souvenirs et des traumatismes de niveau et d'intensité distincts. L'abondante production littéraire, ciménatographique, historiographique que l'on connaît pour la Guerre n'a pas d'équivalent pour le franquisme. Pour partie, on peut expliquer cette absence par le sentiment diffus de culpabilité que de nombreux Espagnols ressentent vis-à-vis d'un régime qui leur offrit la paix, la prospérité et pour beaucoup d'entre eux, l'ascension sociale : le franquisme sociologique se réfugie donc dans une fuite en avant compréhensible, continuant à véhiculer une image très ambiguë de la dictature. $\mathrm{Ce}$ n'était pas la moindre réussite du franquisme que d'avoir partiellement réussi à convaincre d'amples couches sociales que démocratie rimait avec guerre civile.

En somme, j'hérite d'une histoire des mémoires complexe. Mon âge me situe à michemin entre les "enfants" et les "petits-enfants" de la Guerre. Mon statut d'étranger me protège partiellement des enjeux politiques de cette question. A mon sens, il faut bien admettre que le contexte ne détermine pas seulement la production du document mais aussi sa réception et sa lecture dans l'actualité. Autrement dit, suis-je certain qu'à partir de la même source, doté des mêmes instruments de pensée critique et des mêmes paradigmes interprétatifs, mon travail eût été le même il y a 10 ou 20 ans? Suis-je même certain que Les Satrapes de l'Occident m'aurait intéressé? Ne l'aurais-je pas ignoré, tout simplement? Je crois que cette interrogation vaut d'être examinée. Y répondre par la l'affirmative, c'est questionner le statut d'une discipline qui s'est persuadée avoir destin lié aux sciences sociales. C'est admettre que l'objectivation de la mémoire, nécessaire et même salutaire, ne suffit pas à rendre compte de tout. C'est reconnaître enfin que le document n'est pas réductible à une simple archive, qu'il contient une intention dénonciatrice et descriptive qui nous touche et dont on doit rendre compte, à moins de perdre une autre part de nous même, celle du passeur, selon la belle expression de Nicole Lapierre. C'est admettre en un mot la relativité du point de vue de l'historien sur le document.

\section{Le crayon du charpentier.}

\footnotetext{
${ }^{42}$ Créée à la fin de l'année 2000 par Emilio Silva et Santiago Macías, cette association a pour but l'identification des corps demeurant encore dans les fosses communes.

${ }^{43}$ Josep Maria Colomer, La transición a la democracia : el modelo español, Barcelona, Anagrama, 1998, p. 181 .
} 
Dans Le crayon du charpentier, Manuel Ribas raconte l'histoire d'un médecin et militant républicain à Santiago de Compostela, amoureux de la fille d'un cacique local ${ }^{44}$. Arrêté le jour du soulèvement militaire, il ne peut échapper aux griffes de la répression franquiste qui s'abat violemment sur la Galice dès l'été 36. Sans le savoir, il jouit de la protection de l'un de ses geôliers qui est un ami d'enfance de l'amante. La vision binaire classique qui oppose "bons" et "méchants" s'en trouve brouillée. Parmi les prisonniers et ami du médecin, un peintre ne cesse de croquer les scènes de la prison à l'aide d'un crayon de charpentier qu'il a conservé. Contraint par la Phalange d'exécuter le peintre, l'ange-gardien garde sur lui le crayon comme un fétiche qui l'aide à ne pas perdre raison. Aujourd'hui, à la veille de sa mort, l'ancien gardien, maintenant concierge d'un bordel, raconte son histoire à une jeune prostituée immigrée, puis, il meurt. La prostituée, pourtant étrangère à la guerre, récupère le crayon, symbole de la transmission de cette mémoire vive aux générations ultérieures, grâce à l'artifice de la narration. Comme la prostituée de Manuel Ribas, je récupére une histoire qu'il me faut transmettre d'une manière ou d'une autre.

La métaphore du crayon du charpentier oblige l'historien à franchir un pas qui pourrait remettre en cause les fondements de sa discipline. De même que le sociologue a pour souci premier de comprendre la position sociale qu'il occupe par rapport à son objet d'étude, l'historien pourrait se préoccuper de la position temporelle qu'il occupe par rapport au document qu'il lit. Le temps écoulé entre l'archive passé et l'actualité de la lecture historienne n'est pas un temps vide, suspendu, qui ne compterait pas. Ce temps a joué, travaillé, si bien qu'il a modifié profondément le document. La présence - et l'absence du document - dans les différents présents qui s'écoulèrent depuis son écriture sont partie intégrante du document tel que j'en hérite, 60 ans après les faits. Cet héritage eût été nécessairement différent il y 20 ans, de même qu'il le sera dans 20 ans. L'histoire doit intégrer ces jeux de mémoire qui font le document.

$\mathrm{Au}$ fond, histoire et mémoire entretiennent des relations plus complexes que l'histoire de la mémoire a voulu reconnaître. Double intimité : en premier lieu, intimité de deux modes de narration du passé dont la visée diffère : quand l'histoire vise la vérité des faits, la mémoire vise la fidélité. La prétention des historiens à épuiser toute parole sur le passé, c'est-à-dire à sauvergarder un monopole d'interprétation qui légitime en partie leur utilité sociale, n'est plus tenable. De toute façon, nous sommes forcés de reconnaître qu'en dépit des connaissances accumulées, la mémoire continue de suivre son cours comme si de rien n'était. Autrement dit, narrations historique et narration mémorielle n'ont pas nécessairement à coïncider.

En second lieu, entre histoire et mémoire, il y a l'intimité de deux conceptions du passé. Pour l'historien, le passé est révolu, accompli, définitivement mort. Pour la mémoire, le passé est présent ; il est ce qui est encore, ou ce qui n'est plus. Cette autre forme de relation au passé, en somme, s'intéresse davantage à la durée qu'au passé. Le passé n'est pas tant ce qui fut, que le passage du temps. L'histoire-mémoire que j'imagine, ce serait l'histoire du passé pour ce qu'il fut mais aussi, et de manière indissociable, pour ce qu'il n'est plus ou est encore. La mémoire n'y compterait pas pour son contenu, qu'il faut continuer à soupçonner, mais pour sa forme, sa posture vis-à-vis du passé.

\footnotetext{
${ }^{44}$ Manuel Ribas, El lápiz del carpintero, Madrid, Santillana de ediciones, 2001. Le romqn q été adapté à l'écran par Anton Reixa en 2002.
} 
Le manuscrit de Poitiers, décidément, me conduit à remettre en question la position d'extériorité que la raison historienne m'amène naturellement à adopter. Comme une balle de jokari, le devoir de mémoire que ma raison critique semblait avoir définitivement éloigné de moi me revient aujourd'hui avec violence. Quel type d'écriture permettra d'assumer "la part d'ombre" de l'enquêteur? Une écriture qui, dans un constant aller-retour entre passé et présent, rendrait compte des vides de la recherche, des archives brûlées, des vies disparues à jamais, des râtés de la certitude historique. L'écriture d'une histoire lacunaire, attentive au manques, irréparables, et aux absences. Une écriture qui emprunterait à la mémoire le meilleur d'elle-même, une posture au passé, en laissant de côté ses contenus contestables. Une écriture de la présence-absence du passé. "Ce qu'il faut cerner, écrit régine Robin, c'est le travail de la trace en nous".

Entre l'archive dont on feint de croire encore qu'elle contient toute la vérité des faits et le témoignage qui trompe et abuse de notre confiance, il existe un espace pour le document, doublement constitué par son contexte de production, celui du promoteur de mémoire, et de réception, celui de l'historien. L'histoire-mémoire, en réhabilitant le document, retrouve une spécificité disciplinaire peut-être perdue de vue ces derniers temps : celle de l'étude des hommes dans le passé, dans toutes les dimensions de ce dernier. 